\title{
Lingual mucosa graft ureteroplasty for ureteral stricture: a narrative review of the current literature
}

\author{
Kunlin Yang ${ }^{1,2,3}$, Shubo Fan ${ }^{1,2,3},{\text { Zhihua } \mathrm{Li}^{1,2,3}, \text { Hua Guan }}^{1,2,3}$, Peng Zhang ${ }^{4}$, Xuesong Li $^{1,2,3}$, Liqun Zhou ${ }^{1,2,3}$ \\ ${ }^{1}$ Department of Urology, Peking University First Hospital, Beijing, China; ${ }^{2}$ Institute of Urology, Peking University, Beijing, China; ${ }^{3}$ National \\ Urological Cancer Center, Beijing, China; ${ }^{4}$ Department of Urology, Emergency General Hospital, Beijing, China \\ Contributions: (I) Conception and design: X Li, L Zhou; (II) Administrative support: L Zhou, H Guan; (III) Provision of study materials or patients: \\ K Yang, P Zhang; (IV) Collection and assembly of data: S Fan, Z Li, H Guan; (V) Data analysis and interpretation: K Yang; (VI) Manuscript writing: \\ All authors; (VII) Final approval of manuscript: All authors. \\ Correspondence to: Xuesong Li. Department of Urology, Peking University First Hospital, Beijing, China; Institute of Urology, Peking University, \\ Beijing, China; National Urological Cancer Center, No. 8 Xishiku St., Xicheng District, Beijing 100034, China. Email: pineneedle@sina.com.
}

\begin{abstract}
Long proximal or middle ureteral strictures remain challenging conditions. Although ileal replacement of the ureter or renal autotransplantation are possible solutions for these problems, both present complexities and considerable morbidity. In recent years, many urologists have tended to use oral mucosal grafts to repair complex proximal or middle ureteral strictures and thereby avoid ileal replacement and renal autotransplantation. A comprehensive search of oral mucosa graft ureteroplasty and lingual mucosa graft (LMG) ureteroplasty was performed. All literatures must be in English language. Most reports related to oral mucosa graft ureteroplasty focus on buccal mucosa grafts (BMGs). The available data about LMG ureteroplasty are limited. Both LMG and BMG are oral mucosa grafts that have similar histological structures. The success rates of urethroplasty with LMG and BMG are similar. Although there is still no comparative study between LMG and BMG for ureteroplasty, the limited reports show that LMG ureteroplasty is safe and effective for the management of ureteral strictures. This method provides one more option for the management of long proximal and/or middle ureteral strictures. However, the current studies on LMG ureteroplasty have a small sample size and are retrospective. More prospective, multicenter and large sample studies with long-term follow-up results that can further prove the efficacy of LMG ureteroplasty are still needed.
\end{abstract}

Keywords: Lingual mucosa graft (LMG); ureteral stricture; ureteroplasty; ureteral reconstruction

Submitted Nov 25, 2020. Accepted for publication Feb 24, 2021.

doi: 10.21037/apm-20-2339

View this article at: http://dx.doi.org/10.21037/apm-20-2339

\section{Introduction}

Ureteral stricture disease (USD) is defined as narrowing of the lumen of the ureter for a variety of reasons and is mainly attributed to extrinsic or intrinsic factors (1). The common causes include congenital abnormalities, iatrogenic injury from surgery, ureterolithiasis, post radiation therapy, retroperitoneal fibrosis, trauma, infection, and endometriosis (2-4). The management of USD has a widevariety of options that mainly depend on the individual situation. Endoscopic management should be highly selective given that ureteral reconstruction remains the gold-standard treatment for USD (1).

For short ureteral strictures of the proximal or middle ureter, watertight, tension-free and end-to-end anastomosis always represents a feasible first choice. However, long proximal or middle ureteral strictures remain a major challenge. In some complex cases, the cicatricial tissues around the ureter and the segmental diseased ureter should be excised, which results in the failure of simple anastomosis. Traditionally, ileal replacement of the ureter or renal autotransplantation represent common treatments for 
Table 1 The development process of lingual mucosa graft ureteroplasty for ureteral strictures

\begin{tabular}{llll}
\hline Year & Author (Ref.) & Animal experience or clinical application & Laparoscopic or robotic \\
\hline 2012 & Hassan et al. (17) & Animal experience & No \\
2015 & Li et al. (19) & Clinical application & Laparoscopic \\
2016 & Li et al. (20) & & Robotic \\
2018 & Beysens et al. (21) & Clinical application & Laparoscopic + robotic \\
2021 & Fan et al. (22) & Clinical application & Laparoscopic + robotic \\
2021 & Cheng et al. (23) & Clinical application & No \\
2021 & Xu et al. (18) & Animal experience &
\end{tabular}

*, the first report about laparoscopic LMG ureteroplasty in human; ${ }^{* \star}$, the first report about robotic LMG ureteroplasty in human by video form; ${ }^{* *}$, the largest case series about LMG ureteroplasty in human up to now. LMG, lingual mucosa graft.

these conditions. Nevertheless, both options have to face high morbidity rates of complications in addition to their inherent complexities $(5,6)$.

In recent years, an increasing number of urologists have used oral mucosa grafts (OMGs) or appendiceal grafts to facilitate the management of long proximal or middle USDs and to reduce the utility of ileal replacement and renal autotransplantation (7-11). With the usage of onlay repair techniques, the lumen of the narrow ureteral segment can be sufficiently expanded to allow urine to flow through the nonobstructive ureter. Buccal mucosa graft (BMG) ureteroplasty has been reported by many centers and has shown its feasibility and safety (9-11). The lingual mucosa graft (LMG) is a type of OMG that has a histological structure similar to that of BMG $(12,13)$. The success rates of urethroplasty with LMG and BMG are similar (12,14-16). However, no comparative study between LMG and BMG ureteroplasty has been published to date. Very few reports about LMG ureteroplasty have been published (17-23). The Table 1 shows the development process of LMG ureteroplasty for ureteral strictures. In this article, we provided a comprehensive review of LMG ureteroplasty. We present the following article in accordance with the Narrative Review reporting checklist (available at http:// dx.doi.org/10.21037/apm-20-2339).

\section{Methods}

This article reviewed LMG ureteroplasty research up to February 2021. A comprehensive search of OMG ureteroplasty and LMG ureteroplasty was performed using the online database. Studies on LMG ureteroplasty are the focus of. All studies must be in English language. Non-
English language studies were excluded.

\section{Animal experiments}

In 2012, Hassan and Elbakry reported an animal experiment that used LMG to replace the long ureteral defect (17). The study was performed on 9 dogs. LMGs $10 \mathrm{~cm}$ in length and $1-1.5 \mathrm{~cm}$ in width were harvested from the ventrolateral mucosal surface of the tongue. A ureteral defect was simulated by longitudinally excising part of the right ureteral wall approximately $10 \mathrm{~cm}$ in length. Then, the harvested LMG replaced the right ureteral defect as an onlay graft. Furthermore, the area of the anastomosis was covered with omentum. The dog underwent intravenous pyelography (IVP) at the 4th, 8th and 12th weeks. Gross and histological examination of the right ureter and kidney were assessed to evaluate the potency of the reconstructive ureter at the 12th week after all dogs were euthanized. All dogs survived without early and late postoperative complications. IVP showed good drainage of the right kidney without any signs of ureteral stricture or extravasation. Gross and histological examination of the right ureter showed that the LMGs grew well.

In 2021, Xu et al. reported that LMG ureteroplasty is feasible in a Beagle model (18). They randomly divided 12 dogs into 3 groups (the lengths of the ventral ureteral defects were 3, 6 and $10 \mathrm{~cm}$ ). The results showed that one $\mathrm{dog}$ in the long ureteral defect $(10 \mathrm{~cm})$ group developed a mild stricture. The remaining 11 dogs obtained successful repair of the ureteral defect. The duration of experiment was 12 months.

Although the follow-up time of both studies was short, these experiments demonstrated the feasibility and safety of 

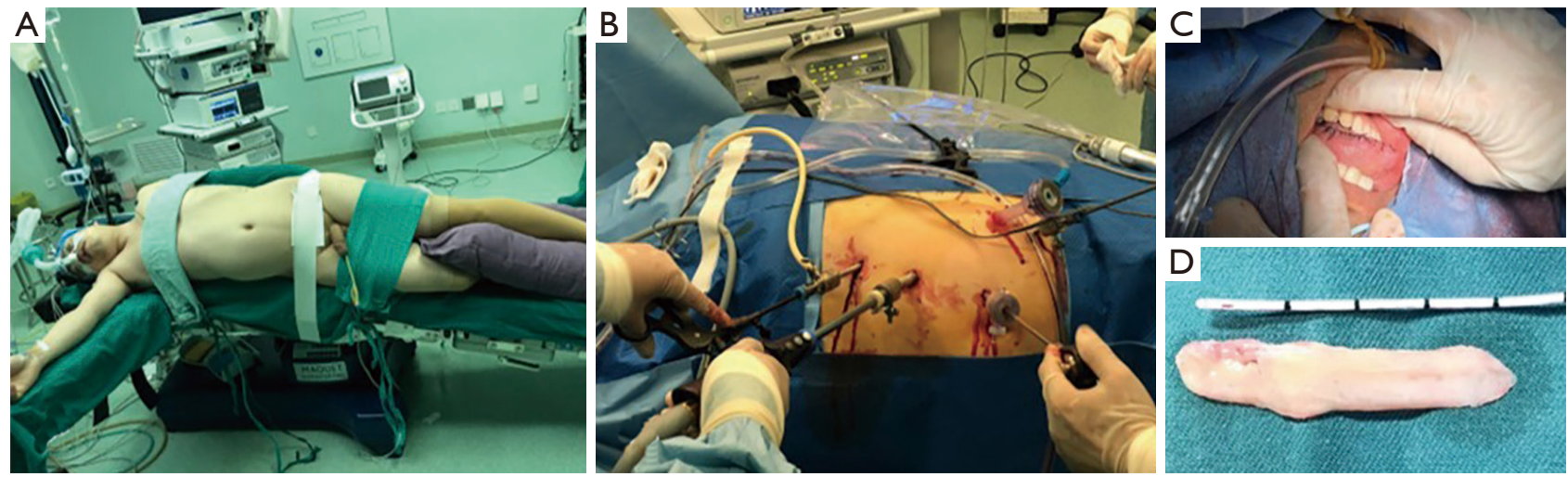

Figure 1 The position of the patient, the trocars' placement and the harvest of LMG. The position of the patient (A) and trocars' placement (B) in laparoscopic LMG ureteroplasty. The donor site of LMG (C) and a harvested LMG (D). (Image courtesy of Xuesong Li, MD). LMG, lingual mucosa graft.

LMG ureteroplasty in a dog model.

\section{Indications for LMG ureteroplasty}

The indications for LMG ureteroplasty are benign long proximal or middle ureteral strictures $(>2 \mathrm{~cm})$ not amenable to primary excision and anastomosis techniques, such as ureteroureterostomy, pyeloplasty or ureterocalicostomy $(19,20,22,23)$. Similar to BMG ureteroplasty, the aim of LMG ureteroplasty is to facilitate the management of complex ureteral strictures and thereby avoid the utilization of ileal replacement and renal autotransplantation.

\section{Laparoscopic LMG ureteroplasty}

Li et al. published their first case of laparoscopic ventral onlay LMG ureteroplasty in 2015 (19). To the best of our knowledge, this is the first case of laparoscopic LMG used in the human body worldwide. A 42-year male patient had a $3-\mathrm{cm}$ proximal ureteral stricture that included $1-\mathrm{cm}$ occlusion. They used a LMG that was $4.6 \mathrm{~cm}$ long and $1.5 \mathrm{~cm}$ wide to repair the stricture. The surgery was performed through three ports: one $10-\mathrm{mm}$ port for the camera, one $5-\mathrm{mm}$ port and one $10-\mathrm{mm}$ port for the surgeon. The epithelium of the graft faced the ureteral lumen. The ventral onlay LMG was performed intermittently using 4-0 Vicryl sutures. No perioperative complications occurred. The patient's pronunciation returned to normal 2 weeks after surgery. The double-J stent was removed 8 weeks after surgery. The ultrasound showed no hydronephrosis 12 weeks after surgery. However, the follow-up time of this case was only 3 months.
Nine months after surgery, Li et al. reported this case again. The glomerular filtration rate of the affected-side kidney recovered from $9.6 \mathrm{~mL} / \mathrm{min}$ preoperatively to $14 \mathrm{~mL} / \mathrm{min}$ at the 6 -month follow-up. The patient had no complaints about the donor site. The computerized tomography scan showed significant relief of the left hydronephrosis (20).

As reported by Fan et al. and Cheng et al. (22,23), laparoscopic LMG ureteroplasty can be performed in selective patients who have indications for LMG ureteroplasty. After general anesthesia, nasal tracheal intubation was inserted. The patient was placed on the affected side in a $45^{\circ}-60^{\circ}$ lateral position (see Figure 1). The four-trocar technique was preferred (see Figure 1). When the surgeon decided to use LMG, an assistant who had been trained for harvesting OMGs prepared to perform the LMG harvest. After the surgeon measured the length of the ureteral stricture or defect, the assistant started to harvest the LMG at a proper length (see Figure 1). The onlay repair of ureteroplasty was generally sufficient (see Figure 2). If the ureter had complete occlusion, segmental excision of the diseased ureter was needed. Then an augmented posterior anastomosis of the ureter plus anterior LMG ureteroplasty was performed. At the end of surgery, the area of anastomosis was wrapped by omentum $(22,23)$. The success rate of LMG ureteroplasty was $100 \%$ with a mean 15.5-month follow-up time (23).

\section{Robotic LMG ureteroplasty}

In 2018, Beysens et al. reported the first case of robotic LMG ureteroplasty in the form of a video (21). The patient 

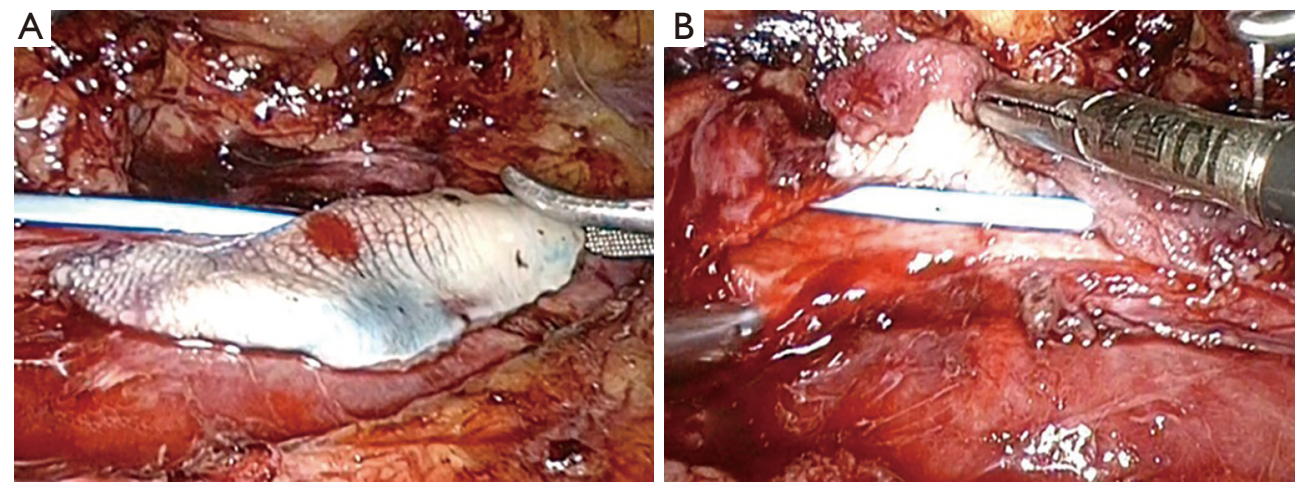

Figure 2 Laparoscopic LMG ureteroplasty. (Image courtesy of Xuesong Li, MD). LMG, lingual mucosa graft.
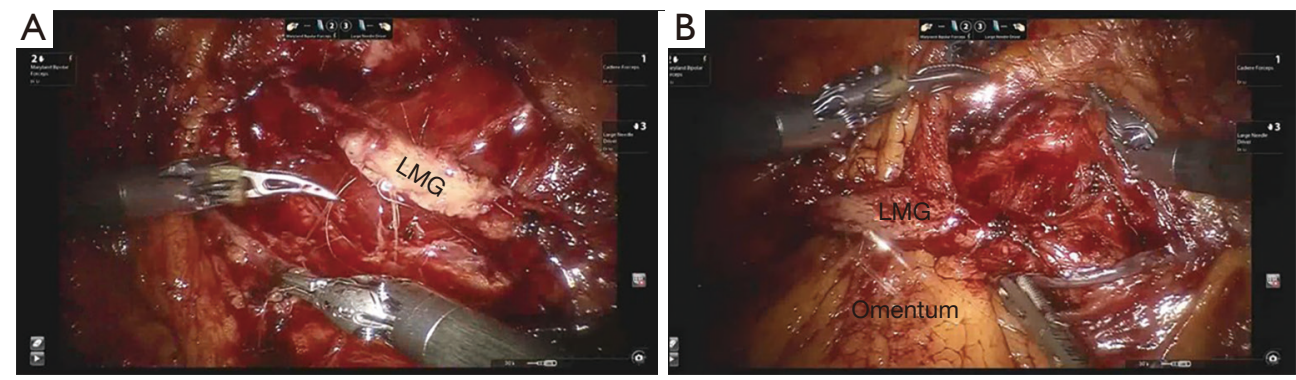

Figure 3 Robotic LMG ureteroplasty (A) and omental flap wrapping the LMG (B). (Image courtesy of Xuesong Li, MD). LMG, lingual mucosa graft.

was a 46-year-old woman with a proximal ureteral stricture $2 \mathrm{~cm}$ in length. The surgical team harvested LMGs that were $4 \mathrm{~cm}$ in length and $1.5 \mathrm{~cm}$ in width. The donor site was not closed. Augmented posterior anastomosis was performed. Anterior onlay LMG was performed using running sutures PDS 5-0. The double-J stent was removed 6 weeks after surgery. Three months after surgery, the patient underwent flexible ureteroscopy for the renal stone, which showed that the mucosa of the graft was intact, widely patent and well vascularized. However, no long-term results about this case were reported.

In 2021, Fan et al. and Cheng et al. reported small case series of robotic LMG ureteroplasty $(22,23)$. Robotic surgery maintains the benefits of minimally invasive surgery and provides the surgeon with special advantages in surgical vision, ureteral dissection and precise anastomosis (see Figure 3). The patient's position (see Figure 1) and the detailed surgical technique were similar to those of laparoscopic LMG ureteroplasty. Cheng et al. reported 5 cases of robotic LMG ureteroplasty, which is the largest case series reported for this technique (23). The success rate of robotic LMG ureteroplasty was $80 \%$ with a mean 8 -month follow-up time. One patient with 6 months of follow-up suffered from aggravated hydronephrosis and recurrent flank pain after double-J stent removal. A second nephrostomy was performed. Therefore, they judged this case as a failure when they wrote their article.

\section{Limitations of the current literature}

The limitations of the current literature about LMG ureteroplasty are obvious. The limitations include are small sample sizes, single-center studies, short follow-up times and the retrospective nature of these studies. We still lack studies with large sample sizes performed at multiple centers with long-term follow-up.

In addition, there is still no comparative study between BMG and LMG ureteroplasty to demonstrate the more effective technique. Most studies on OMG urethroplasty demonstrated that LMG was not inferior to BMG $(12,15,16)$. Kumar et al. found that LMG urethroplasty had the same results as BMG urethroplasty with lower 
donor site morbidity (16). In a systematic review of LMG urethroplasty, Abrate et al. concluded that LMG should be the first choice for urethral strictures with fewer oral complications (24). However, Sharma et al. noted that postoperative morbidity and long-term changes in speech after LMG ureteroplasty for strictures $>7 \mathrm{~cm}$ made LMG the second choice (14). Controversial issues mainly focus on donor site morbidity and oral complications.

Therefore, it is too early to conclude that LMG ureteroplasty has equivalent efficacy to BMG ureteroplasty.

There are many limitations to designing a comparative study between BMG and LMG ureteroplasty. Long proximal ureteral strictures are relatively uncommon, and it is very difficult to include a large series of patients in a single center. The multitude of modalities used to evaluate stricture recurrence and surgical success make it difficult to perform multiple-center studies (4).

Moreover, we do not know the maximal length of the ureteral stricture used for treatment with OMG ureteroplasty. In the literature, the maximal length of the ureteral stricture in humans treated by BMG ureteroplasty ranged from 5 to $8 \mathrm{~cm}(9-11,25)$.

\section{Further directions}

Ideally, prospective, multicenter and large sample studies with long-term follow-up results would provide more data about the outcomes of LMG ureteroplasty. With the increasing use of robotics in ureteral reconstructive surgeries, robotic LMG ureteroplasty may become more common. LMG ureteroplasty may offer one more choice for the management of long proximal and/or middle ureteral strictures.

\section{Conclusions}

LMG ureteroplasty seems to be a safe and feasible technique that provides one additional option for the management of long proximal and/or middle ureteral strictures. However, more prospective, large sample and multicenter studies with long-term follow-up results are still needed.

\section{Acknowledgments}

We are appreciated to the American Journal Experts which provides the service of English language editing.

Funding: None.

\section{Footnote}

Reporting Checklist: The authors have completed the Narrative Review reporting checklist. Available at http:// dx.doi.org/10.21037/apm-20-2339

Conflicts of Interest: All authors have completed the ICMJE uniform disclosure form (available at http://dx.doi. org/10.21037/apm-20-2339). The authors have no conflicts of interest to declare.

Ethical Statement: The authors are accountable for all aspects of the work in ensuring that questions related to the accuracy or integrity of any part of the work are appropriately investigated and resolved.

Open Access Statement: This is an Open Access article distributed in accordance with the Creative Commons Attribution-NonCommercial-NoDerivs 4.0 International License (CC BY-NC-ND 4.0), which permits the noncommercial replication and distribution of the article with the strict proviso that no changes or edits are made and the original work is properly cited (including links to both the formal publication through the relevant DOI and the license). See: https://creativecommons.org/licenses/by-nc-nd/4.0/.

\section{References}

1. Lucas JW, Ghiraldi E, Ellis J, et al. Endoscopic Management of Ureteral Strictures: an Update. Curr Urol Rep 2018;19:24.

2. Klap J, Phe V, Chartier-Kastler E, et al. Aetiology and management of iatrogenic injury of the ureter: a review. Prog Urol 2012;22:913-9.

3. Vrettos A, Prasinou M, Frymann R. Ureteral endometriosis: an uncommon cause of ureteral stricture. Quant Imaging Med Surg 2016;6:231-2.

4. Lee Z, Keehn AY, Sterling ME, et al. A Review of Buccal Mucosa Graft Ureteroplasty. Curr Urol Rep 2018;19:23.

5. Zhong W, Du Y, Yang K, et al. Ileal Ureter Replacement Combined With Boari Flap-Psoas Hitch to Treat Full-Length Ureteral Defects: Technique and Initial Experience. Urology 2017;108:201-6.

6. Zhong W, Hong P, Ding G, et al. Technical considerations and outcomes for ileal ureter replacement: a retrospective study in China. Bmc Surg 2019;19:9.

7. Wang J, Xiong S, Fan S, et al. Appendiceal onlay flap 
ureteroplasty for the treatment of complex ureteral strictures: initial experience of nine patients. J Endourol 2020;34:874-81.

8. Waldorf B, Lee Z, Kidd L, et al. Robotic Buccal Ureteroplasty: a Review of the Current Literature. Curr Urol Rep 2017;18:40.

9. Lee Z, Waldorf BT, Cho EY, et al. Robotic Ureteroplasty with Buccal Mucosa Graft for the Management of Complex Ureteral Strictures. J Urol 2017;198:1430-5.

10. Badawy AA, Abolyosr A, Saleem MD, et al. Buccal mucosa graft for ureteral stricture substitution: initial experience. Urology 2010;76:971-5.

11. Zhao LC, Weinberg AC, Lee Z, et al. Robotic Ureteral Reconstruction Using Buccal Mucosa Grafts: A Multiinstitutional Experience. Eur Urol 2018;73:419-26.

12. Lumen N, Vierstraete-Verlinde S, Oosterlinck W, et al. Buccal Versus Lingual Mucosa Graft in Anterior Urethroplasty: A Prospective Comparison of Surgical Outcome and Donor Site Morbidity. J Urol 2016;195:112-7.

13. Markiewicz MR, Margarone JE, Barbagli G, et al. Oral Mucosa Harvest: An Overview of Anatomic and Biologic Considerations. EAU-EBU Update Series 2007;5:179-87.

14. Sharma AK, Chandrashekar R, Keshavamurthy R, et al. Lingual versus buccal mucosa graft urethroplasty for anterior urethral stricture: a prospective comparative analysis. Int J Urol 2013;20:1199-203.

15. Pal DK, Gupta DK, Ghosh B, et al. A comparative study of lingual mucosal graft urethroplasty with buccal mucosal graft urethroplasty in urethral stricture disease: An institutional experience. Urol Ann 2016;8:157-62.

16. Kumar A, Das SK, Trivedi S, et al. Substitution urethroplasty for anterior urethral strictures: buccal versus lingual mucosal graft. Urol Int 2010;84:78-83.

17. Hassan MM, Elbakry AM. Lingual Mucosal Graft as a Long Segment Ureteric Replacement: An Experimental Study in Dogs. Journal of American Science 2012;8:256-61.

18. Xu Y, Sun L, Pan Q, et al. A new technique for ureteral reconstruction using lingual mucosa grafts in a beagle model. Int Urol Nephrol 2021;53:83-9.

19. Li B, Xu Y, Hai B, et al. Laparoscopic ventral onlay lingual mucosal graft ureteroplasty for repair of proximal ureteral stricture: initial experience and short-term outcome. Journal of Clinical Urology 2015;30:869-71.

20. Li B, Xu Y, Hai B, et al. Laparoscopic onlay lingual mucosal graft ureteroplasty for proximal ureteral stricture: initial experience and 9-month follow-up. Int Urol Nephrol 2016;48:1275-9.

21. Beysens M, De Groote R, Van Haute C, et al. Robotic lingual mucosal onlay graft ureteroplasty for proximal ureteral stricture. Eur Urol Suppl 2018;17:e1935.

22. Fan S, Yin L, Yang K, et al. Posteriorly Augmented Anastomotic Ureteroplasty with Lingual Mucosal Onlay Grafts for Long Proximal Ureteral Strictures: 10 Cases of Experience. J Endourol 2021;35:192-9.

23. Cheng S, Fan S, Wang J, et al. Laparoscopic and robotic ureteroplasty using onlay flap or graft for the management of long proximal or middle ureteral strictures: our experience and strategy. Int Urol Nephrol 2021;53:479-88.

24. Abrate A, Gregori A, Simonato A. Lingual mucosal graft urethroplasty 12 years later: Systematic review and metaanalysis. Asian J Urol 2019;6:230-41.

25. Zhao LC, Yamaguchi Y, Bryk DJ, et al. Robot-Assisted Ureteral Reconstruction Using Buccal Mucosa. Urology 2015;86:634-8.
Cite this article as: Yang K, Fan S, Li Z, Guan H, Zhang P, Li X, Zhou L. Lingual mucosa graft ureteroplasty for ureteral stricture: a narrative review of the current literature. Ann Palliat Med 2021;10(4):4840-4845. doi: 10.21037/apm-20-2339 